\title{
Functorial Models of Spatio-Temporal Landscape Field of the Earth: Methods of Comparative-Geographical Studies Based on Data of Remote Sensing
}

\author{
E.A. Istomina* \\ V.B.Sochava Institute of Geography SB RAS, Irkutsk, Russia
}

\begin{abstract}
This paper suggests models of the landscape complexes based on the theory of complex systems and category-functorial approach. With the use of the space Landsat images the existence of the Earth's landscape field is proved and criteria of its parameter characterization is suggested. The unity of spatiotemporal changes of the landscape units is substantiated.
\end{abstract}

Key words: geographical complex, comparative geography, landscape field, theory of categories and functors, landscape mapping, spatial and temporal connections

AMS subject classification: 91D20

\section{Introduction}

\subsection{Origin of the problem}

The majority of geographers of the Soviet scientific schools consider modelling of complex geographical objects and processes difficult because of the need of consideration of a large number of factors, and the results obtained trivial [22]. The solution of this problem depends on the correct selection of mathematical tools. For model making of spatial structure of the Earth's landscape envelope it is necessary to create a structure, which takes into account different interrelations between components and units of geographical complexes, transformation of these interrelations, and hierarchy of geocomplexes. The theory of categories and functors is such a tool. Unlike the set theory, the category theory includes not only the sets (objects), but also mappings of objects and mappings of mappings [25]. Therefore, the category theory allows the unity of the geographical envelope to be modelled - a system of its connectedness geospheres at different levels of their

*E-mail: elena@irigs.irk.ru 
organization. Using this approach, there is no need to take into account all characteristics of the geographical envelope, as they reflect each other. An incomplete complex becomes a model of the complete one.

\subsection{Geographical background}

Although a large number of mathematical publications is devoted to the category theory and they deal with the modelling of interconnected environmental systems, which are transformed in space and time $[6,33]$, there are few examples on the application of this theory in natural sciences. There are well-known publications on the category-functorial modelling in biology [25, 26, 27, 4, 37]. In geography, modelling of this kind is directly connected with the modelling of a landscapegeographical field, which is formed on the conceptions of polystructural properties of the landscape envelope, landscape space as a quantum continuum [32].

There are different approaches to the modelling of geographical fields. The simplest and most common variant is the creation of isolinear maps with characteristics of geographical phenomena (relief, temperature, air humidity, precipitation, etc.). The mapping of fields of interrelation between certain components is a more complicated approach $[5,20]$. This approach to the landscape field corresponds to the viewpoint of D.L. Armand [2], who regards the field as "a connection source in systems". Modelling of this field implies both vertical and horizontal (spatial) connections in geosystems, as well as all connections in total, which requires a category-functorial tool. In spite of the research carried out in this field [1, 2, 3, 31], at present there is no formalized theory reflecting general laws on the geographical envelope formation.

\section{Model and methods}

Models of the Earth's landscape fields are constructed within the framework of axioms of the theory of composite complex systems $[8,9]$ formulated in terms of the theory of categories and functors. Theories correspond to the integrated approach in geography used to investigate the laws of synthesis of different-quality parts into a common territorial object. The "landscape complex" is the central term. Objects, morphisms, categories, functors, and toposes are the basic system concepts in the description of landscape complexes.

By a part of a landscape complex $\left(X_{i}\right)$ is meant any $(i$-th) object or a set of objects of differentquality elements in the Earth's landscape sphere: a landscape component or a combination of components, facies unit, their combination, etc. Several sets are present simultaneously at the same place: a system soil-vegetation, water-air; a system of facies units of the same stow, a set of facies units belonging to one group, etc. This approach corresponds to the principle of polystructural properties of the terrestrial landscapes.

Information connection of different parts of a complex is regarded as the comparison ratio (functional connection) and is specified by the morphism $F_{i j}: X_{i} \rightarrow X_{j}$ of objects - by their functional similarity. The comparison of two sets $X_{i}, X_{j}$ is a new set $\Delta X_{i j}=X_{j} / X_{j}$ (out of the set $\Delta X$ ), which shows how much the $j$-th set differs from the $i$-th. The set of all possible 
morphisms $F_{i j}$ is combined into the universal set $F$ and forms a class of morphisms, whereas the set of all objects $X_{i}$ - into $X$ forming a class of objects. Category is a system of similarity of several objects (the system of functional connections of parts of the landscape whole): $X_{i} \rightarrow X_{j} \rightarrow X_{k}$.

The similarity standard $I=[0,1]$ is generated, i.e. it is a metrized, linearly ordered, continuous set of points (values) bounded above and below. One-to-one correspondence of objects, morphisms, comparisons and standards is designated by the ratio $(\leftrightarrow)$, for example, $X \leftrightarrow I$ means that any object out of the set $X$ unambiguously corresponds to a definite value out of the segment [0,1] (a value out of $I$ ): $X(I)=X_{i}$ is a concrete object out of $X$ with the index $i=I$. There is a special concept "topos" in the theory of mathematical categories, which establishes a correspondence between objects-categories and their values. This concept also has a geographical interpretation. In Greek, it initially meant unity (connectedness) and space [21].

These concepts provide a basis for the formulation of axioms of the theory of composite complex systems [8], which imply the comparison of various objects and morphisms with the standard of the order and with one another:

$$
\text { 1) } X \leftrightarrow I ; 2) F \leftrightarrow I ; 3) \Delta X_{i j} \leftrightarrow F_{i j}
$$

Axioms postulate that 1 ) any objects and categories are interrelated (there is a universal connection); and 2) a morphism $F_{i} \leftrightarrow X_{i}$ corresponds to every object. As a result, all objects, morphisms and values form a universal similarity system:

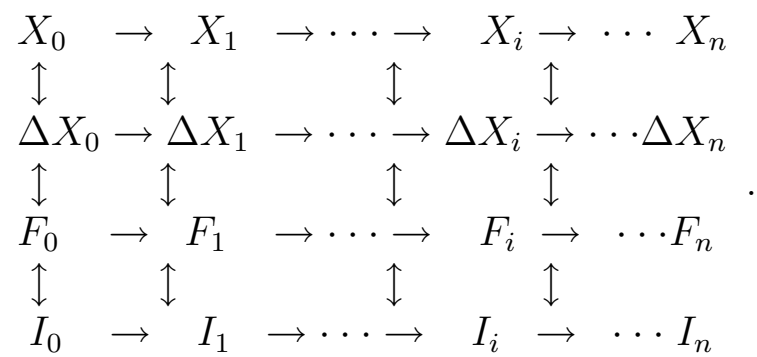

The series in (2.1) are bounded by values below $I_{0}=0$ and above $I_{n}=1$. It follows from (2.1), that all objects $X_{i} \in X$ and morphisms $F_{i} \in F$ are linearly ordered and metrized: $X_{i}=$ $X\left(I_{i}\right), F_{i}=F\left(I_{i}\right), I_{i} \in I$, where $I_{i}$ is a measure indicator (individual value, index) of an object $X_{i}$ and a morphism $F_{i}$. A knowledge of indices of the particular objects allows them to be identified. There is a continuous uniparametric (in $I_{i}$ ) transformation of an object to the object $X\left(I_{i}\right) \rightarrow$ $X\left(I_{j}\right)$ (structural similarity, homology), and of a morphism to the morphism $F\left(I_{i}\right) \rightarrow F\left(I_{j}\right)$ (functional similarity, homotopy). Hence, all structures of the composite system are homologically equivalent, as well as its all functional connections are homotopically equivalent. They differ only in the index value from the segment $[0,1]$ corresponding to these structures and connections, in the homological-homotopical parameter.

Category is an inductive system, i.e. the information is transmitted from one object to another via functional (informational) connections (morphisms). Principles of landscape indication and that of other types are based on this quality: a knowledge of the properties of one component is derived from a knowledge of other components and the landscape in general. Any real category is a fragment of the universal category (the upper row from (2.1)), i.e. it is composed of the concrete 
set of parts, e.g. a landscape is composed by compositions - facies units or stows (polystructural properties). Hence, the definition of the complex model is the following: it is any category satisfying the similarity relations (2.1), i.e. a complex of information exchange is a linearly ordered, bounded, metrized, inductive, and homological-homotopic system [17]. A mathematical topos is a formal model of a geographical complex.

Similarity of the two $k$-th $\left(F_{k i j}: X_{k i} \rightarrow X_{k j}\right)$ and $l$-th $\left(F_{l i j}: X_{l i} \rightarrow X_{l j}\right)$ or several categories is a functor. The model of their connections is specified by a diagram:

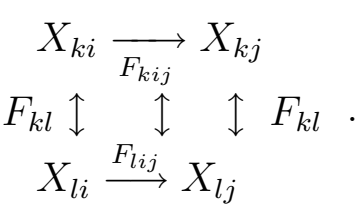

Model (2.2) realizes a principle of the homological and homotopic similarity of differentquality parts and functions, which is the basis for complexing. A functor transforms objects of the category $k$ into objects of the categoryl, morphisms of the category $k$ into morphisms of the category $l$. Hierarchy of the complexes (connection of connectedness) is revealed via a functor.

In addition, model (2.2) shows that there is a rule of the information exchange which is stated in the law of the functional connection type conservation between structures and functions of a landscape. This ubiquitous information exchange forms a functorial field of interaction of the landscape complexes, by which is meant a set of functors of the geographical envelope. On the basis of this set, the laws of integration (2.1) are realized - the laws of structural-functional similarity, which function differently at its each point (a homogeneous domain). The basic principle of the comparative-geographical method is realized in this definition: everything in the geographical envelope is the same up to some transformations (morphisms). Therein lies the basic idea, the usefulness and competitive ability of the geographical knowledge aimed at comparing the differing, at transforming the known into unknown, and based on the category-functorial approach [17].

Geographical complexes traditionally vary in their vertical (intercomponent), spatial and temporal structural-functional order. However, there are no spatial and temporal parameters in axioms (2.1) from the viewpoint of their traditional understanding. Spatial and temporal changes obey some general patterns of integration (2.1). Moreover, there are no principal differences in the similarity of components of one landscape complex, the similarity of complexes from different time intervals and from different space points. Such an approach corresponds to the ergodic Boltzmann principle known in geography, which allows for the dynamics of a geographical complex from its changes in space. There is a specific spatiotemporal functorial landscape field, which needs investigating.

Information that is necessary for the solution of this problem must possess special properties: it must be parametrized, i.e. must vary continuously across physical space-time or in any other multidimensional space of independent attributes, i.e. it must be continual in many parameters and possess partial derivatives for each attribute. Therefore, space-acquired images are used for studying a landscape field, which are an objective spatiotemporal continuous mapping of the structure of landscape complexes and properties inherent in them. Each imaging channel synthesizes a definite set of the properties of landscape components and integral images of these components 
are compared, thus making it possible to indirectly judge the character of connection between the parts of landscape complexes.

The functor existence of a landscape field is substantiated at the first stage. The presence of such functors (interrelation between the landscape envelope parts) may be indicative of the landscape field existence at this place or, in other words, of its "density". The field "density" is supposed to fall on the boundaries of landscape units and in the disturbed (transitional) complexes. A definite type of the category morphisms and their parameters is defined at the second stage.

\subsection{Calculation technique of Jacobian determinant.}

The Jacobian determinant $D[19,36]$ and space-acquired images are used to substantiate the existence of a landscape field, i.e. to reveal intra-systemic and external functional connections of the landscape complexes (morphisms, categories, and functors).

The brightness characteristics of the images are specified parametrically, whereare spatial coordinates of an element of the scan geoimage (pixel) and time of observation. The Jacobian determinant for these variables is

$$
D=\left|\begin{array}{lll}
\frac{\partial x_{1}}{\partial x} & \frac{\partial x_{1}}{\partial y} & \frac{\partial x_{1}}{\partial t} \\
\frac{\partial x_{2}}{\partial x} & \frac{\partial x_{2}}{\partial y} & \frac{\partial x_{2}}{\partial t} \\
\frac{\partial x_{3}}{\partial x} & \frac{\partial x_{3}}{\partial y} & \frac{\partial x_{3}}{\partial t}
\end{array}\right|
$$

Several multichannel, different-time or remote space images are compared with the Jacobian determinant. When $D=0$, there is an interrelation between the pixel brightness of different images, and when $D \neq 0$, there is no interrelation. This method makes it possible to differentiate homogeneous areas from the types of functional connections of element characteristics of geoimages separated by point boundaries. Moreover, it is sufficient that no matter in what way (within which type of a model) this interrelation is carried out: the Jacobian determinant displays the presence or absence of the interrelation for all models of the object description. In its turn, the interrelation presence helps fix the existence of a landscape field as a system of the interrelation between components and territorial units of a landscape.

The method of the Jacobian determinant makes it possible to reveal domains of the interrelation presence-absence. However, it does not allow the parameters of this interrelation to be defined. The method of congruent modelling is used for the solution of this problem.

\subsection{Congruent modelling.}

According to basic axioms (2.1), the value from $I$ unambiguously corresponds to each function (morphism) $F_{i}$, i.e., these functions are one-parameter functions. Therefore, in case of linear and linearized functions these coefficients should be functionally dependent. It is possible when functions form congruence.

Congruence is of special importance for the theory of integration. It is necessary for understanding of quantitative regularities of interrelation and their adjustment to the locations (situa- 
tions). Congruence, in a common sense, corresponds to the rule of preservation of the mapping properties. For example, a line in the linear transformations turns into a line.

Linear congruence (a term from analytical geometry) is formed by a bundle of lines meeting at one point (a node of congruence) (Fig. 1). On a plane, it is specified by an equation

$$
y=a x+b
$$

Congruence is formed, when coefficients $a$ and $b$ of different linear dependences are linearly interrelated $b=-x_{0} a+y_{0}$, regarding the bundle lines as one-parametric dependences (on $a$ ), which is represented in a general law of connection

$$
y-y_{0}=a\left(x-x_{0}\right)
$$

Coordinates of the bundle centre may be regarded as the beginning of coordinates $(0,0)$. All lines of the bundle are similar in the sense that one line can be changed into another by turning through a certain angle. A slope ratio of the line, i..e. the coefficient $a$ of the definite linear dependence, can serve as a similarity parameter.

In other words, function (2.5) corresponds to the category, whose objects are linear dependences (2.4). For example, rank distributions of occurrence of different brightness pixels of a Landsat image are described within the area of each facies on the landscape map of Valley Marta (Pribaikalye) in the semi-logarithmic scale using linear dependences (2.4), which form the congruence (2.5) for one facies class. In this case, a facies, which is represented by one line in the bundle (Fig. 1) and characterized by the definite coefficient $a_{i}$, corresponds to an object. Congruence (2.5) corresponds to a category (in this case, to a facies class) and describes interrelations between category objects (facies). Since each object-facies is characterized by a certain value (coefficient a), a topos (2.5) is a category.

It is obvious that all congruencies with different position of the nodes $\left(x_{0}, y_{0}\right)$ are structurally similar. If the congruence nodes are located in one line-directrix $\left(y_{0}=\alpha x_{0}+\beta\right)$, the lines of all congruencies are ordered into a linear complex (Fig. 2)

$$
y=a\left(x-x_{0}\right)+\alpha x_{0}+\beta
$$

In this ratio, there are already two control parameters $a$ and $x_{0}$ of different levels of generalization. The linear complex corresponds to a functor connecting objects and morphisms of different categories. In the example cited above, a set of facies classes of one landscape corresponds to a functor.

Equation of linear congruence (2.5) corresponds to a set of lines - tangential at the point $\left(x_{0}, y_{0}\right)$ to a generalized function $y=y(z)$ of the impact of the environmental factors $z=$ $\left(z_{1}, z_{2}, \ldots, z_{j}, \ldots, z_{m}\right)$, where each factor $z_{j}=z_{j}(\xi)$ depends on the location coordinates and time $\xi=\left(x_{\xi}, y_{\xi}, z_{\xi}, t_{\xi}\right)$. A variable value $x$ also belongs to the set $z$. Equation of tangent (2.5) satisfies the conditions $a=\frac{\partial y}{\partial x}$. In a general case, this variable depends on the variable values of the set $z$, including $x$, which imparts significant nonlinearity to equation (2.5).

During geographical studies of unique local phenomena, one fails to define an evident type of functions $y=y(z)$, which reveal the morphisms $F$. Therefore, a function local approximation 
(2.5) - a tangent has to be used. Taking into account that coefficient $a$ is a variable value and a function of many variables, tangent (2.5) becomes the exact model of the dependence $y=y(z)$, whereas the parameter $a=a(\xi)$ changes within location and time. To calculate tangents, it is usually enough to have a small data sample, which allows to estimate parameters of equation (2.5) from the limited set of observation data using statistical methods.

Congruence of tangents (2.5), related to the requirement of crossing the lines at one point $\left(x_{0}, y_{0}\right)$, is not a compulsory condition for tangential lines of the function. Since the parameter $a=a(\xi)$ describes a geographical situation (local environmental conditions) and $x$ and $y$ are characteristics of the objects, the availability of congruent dependence (2.5) in different geographical situations and objects suggests the existence of some fundamental regularity, which combines situations and objects and defines their uniformity. Correspondence of the category-functorial approach makes it possible to name this fundamental regularity as a functorial landscape field.

Therefore, a search of functors of a landscape field reduces, in many respects, to the revealing of functional congruencies - complex functions with attributes of the system similarity.

\section{Results}

A variety of aspects of a functorial landscape field are under studies: intercomponent, spatial and temporal connections, as well as spatiotemporal interrelations between parts of a landscape.

\subsection{Modelling of intercomponent relationships.}

To analyze intercomponent morphisms, two multichannel images are processed with the Jacobian determinant (Fig. 3, a, b).

In this case, the Jacobian determinant is calculated from a simplified scheme

$$
D=\left|\begin{array}{ll}
\frac{\partial x_{1}}{\partial x} & \frac{\partial x_{2}}{\partial x} \\
\frac{\partial x_{1}}{\partial y} & \frac{\partial x_{2}}{\partial y}
\end{array}\right|
$$

There are pair interconnections between brightness characteristics of the two channels. The Jacobian determinant is calculated with numerical methods based on raster space-acquired images. Partial derivatives were transformed into a discrete form $-\frac{\partial x_{i}}{\partial x} \approx \frac{\Delta x_{i}}{\Delta x}$, where $\Delta x_{i}$ is a change of brightness characteristic $x_{i}$ at changing a coordinate $x$ into $\Delta x$, which is equal to pixel size (assumed as a distance unit). Therefore, a partial derivative on spatial coordinates equals the difference of brightness values of the neighbouring pixels.

The points of the presence of connection appear as light domains (Fig. 3, c), which form functionally homogenous units (FHU), and the points of no connection (black dots in Fig. 3, c), belonging to FHU boundaries, where a type of one unit turns into a connection type of another unit (Istomina, 2002). The appearance of FHU proves the existence of configurations (complexes) of different components $F_{i j}: X_{i} \rightarrow X_{j}$ (see (2.2)), where $F_{i j}$ is a function of connection between $X_{i}$ and $X_{j}$ within the FHU boundaries. 
The Jacobian determinant takes zero value equals also in those domains, where at least one variable $x_{i}$ remains constant. One may assume that all FHU marked on the image belong to this case. Fig. 4 shows frequency distribution of pixel occurrence of different brightness within FHU in Fig. 4. As illustrated in this figure, the brightness values in the image change in each analyzed channel. Therefore, units with the constant brightness value, which are also present on the territory, are only a particular case of FHU.

A similar result of processing is obtained for a great variety of domains. Territories experiencing anthropogenic influence are covered with boundaries more densely than natural landscapes. Therefore, this method works worse at the strongly disturbed sites of the territory (agricultural territories, felling sites, settlements, etc.). In this case, however, the method can be used for estimation of the disturbance extent of the landscape complexes caused by anthropogenic impact or other activities.

Boundaries of higher and lower hierarchic levels can be differentiated using the Jacobian determinant: values close to zero - internal domains of the units; values comparatively little different from zero - boundaries of low level, corresponding to the image resolution; values much different from zero - boundaries of high level.

The Jacobian determinant makes it possible to pass to a local analysis of geoimages, where the methods of mathematical analysis, including numerical algorithms reflecting an individual character of the geographical reality are employed rather than the methods of statistical processing.

Besides the justification of the existence of a landscape field, the Jacobian determinant allows the structure of a functorial field to be singled out in the form of boundaries of the landscape complexes of different level: boundaries of the level of facies and districts are singled out on the images with a resolution of 10-20 m, on those of $50 \mathrm{~m}-$ a class of facies and locales. The results obtained can be used in the segmentation of images and construction of landscape maps.

The field structure obtained represents one-type dependence between components, which is disturbed on facies boundaries at transition from facies to facies, as each facies should have its own function of connection $[8,36]$. Thus, a facies is represented as a functionally homogenous area, whereas a geographical space differs in a functional characteristic. In other words, a geographical space is continual in its properties but discrete in the character of their connections. Functional criteria of allocation of linear boundaries of geographical systems add a new quality to the landscape studies, when their connections are differentiated rather than component properties. Since the connections identified from criterion $D$ do not depend on the form of the landscape structure interpretation (a subject of research, a pattern of data presentation), a functional structure of a landscape should be invariant to scientific approaches and methods of typification and differentiation of geographical systems. Moreover, the classification (legend to a map) of geosystem and geocomplexes should be integrated and differ only in character of interpretations of each classification position, which is realized in a thematic map of the appropriate content [24].

\subsection{Modelling of spatial and time relationships.}

The Jacobian method was also used for studying relationships of different landscape units (categories) within a distance (a spatial functor) and time (a time functor). Landsat TM images of 
Pribaikalye were studied: 3 images of the Primorsky Ridge territory of different time) and China (the Kunlun Mountains of Province Qinghai).

Three different techniques were used to develop images:

1 Images of different spectrum channels on the territory of the Primorsky Ridge. Relationships were estimated in red and short-ranged infrared channels of the spectrum (Fig. 5 c). The results of image processing using the combination of different channels demonstrate similar form of boundaries. It means that there are stable intercomponent relationships within units (functional homogeneity), i.e., component landscape complexes-categories.

2 Images of red channel on the territory of the Primorsky Ridge taken at different time (Fig. 5 c). In this case boundaries are also revealed which coincide with boundaries in images of different channels. As shown in the first variant of processing, there are interrelations of different components, i.e., there is category $k$ of one time moment and category $l$ of another time moment (see (2.2)). The results of the current processing (see fig. 5 c) show the existence of time (diachronous) functors (2.2) being a peculiar feature of landscape field.

3 Images with significant spatial displacement of the Primorsky Ridge territory and the Kunlun Mounatin system (Fig. 5 d). Boundaries connecting different parts of mountainous territories are distinguished: there is a functional link among characteristics of these territories which is disturbed at the boundary. It means that one image regarding another contains information necessary for identification of homogenous and heterogeneous structures. The pattern of boundaries is more complicated because natural boundaries of two different fragments of the image are overlapped. Hence, there are relationships among different functional homogeneous units situated at a long distance, i.e., spatial functors which form the structure of landscape field. There are morphisms of parts of different complexes $F_{k l}: X_{k i} \rightarrow X_{l i}$ (see (2.2)). Hence, there are refelctions of configurations (2.2) when relationships $F_{k i j}$ of parts of different complexes turn into one another $F_{k i j} \rightarrow F_{l i j}$, i.e., information exchange and exchange of matter and energy exist among units of different distance.

Therefore, there are categories the objects of which are components of landscape and different time and spatial sections of landscapes reflected in brightness characteristics of images of different channels, territories and time of survey. Morphisms of these categories are represented by an unknown function which, on the one hand, is preserved and, on the other hand, is transformed from place to place according to an unknown rule. This is one function (one-type function with the accuracy of equivalent transformations) which should be found.

\subsection{Congruent modelling of spatio-temporal changes.}

The method of congruent modelling (see 2.2) is used for defining the concrete parameters of spatiotemporal functors.

A landscape-typological map of the facies level of the Marta valleydale (Pribaikalye) is used as an information basis [13]. The map was compiled on factorial-dynamic principles [39] based 
on the space images. The territory is located on the interface of steppe and taiga types of the natural environment [24] and represented by mountain-taiga pine and larch forests and steppe communities.

Three different-time space Landsat TM images are analyzed (August, September and November, 2006). Seasonal dynamics, observed on images, corresponds to the changes of geocomplexes at the level of a facies class. Therefore, the changeability of parameters of the homotopic similarity of a class of sublithomorphic exposure facies (pine-larch forests) is under studies. The native facies of this series is represented by pine and larch-pine rhododendron forests with aspen and mountainous dark-grey forest thick soils of the broad watershed surfaces and terraces.

The parameters $a$ and $b$ of the twenty-one rank distribution ${ }^{\dagger}$ of pixels of different brightness were identified from the occurrence for the areas of 7 facies at 3 time periods, which is represented in semi-logarithmic scale as

$$
y=a_{i j} x+b_{i j}
$$

with a correlation coefficient $R$, where $y$ is a logarithm of pixel occurrence of a certain rank, $x-\mathrm{a}$ rank, $i$ - a facies number, and $j$ - survey time. If the coefficients ratio (3.2) are linearly dependent on

$$
a_{i j}=c b_{i j}+c_{0}
$$

with high $R$, the equations studied belong to one congruence (a bundle of lines), whereas the objects described by these ratios form a category.

Rof ratio (3.3) for all distributions studied equals 0.46 . Therefore, all distributions do not belong to one congruence. However, the distributions for each facies (with fixed $j$ ) at different time form a congruence. $R$ for (3.3) ranges between 0.94 and 0.99 for different facies. Such ratios for different $i$ form a linear complex-functor (a set of congruence whose centres are in one line), which is supported by a linear connection $c_{i}=13.9 c_{0 i}-0.3, R=0.94$.

The same scheme was used for the calculations of other facies class of the map mentioned and similar results were obtained. It means that temporal and spatial changes of geocomplexes occur within the subsystems (categories) of an integrated system (functor). With the use of the models offered there is a possibility of transition from one time interval to the other within one facies, as well as from a time series of one facies to that of another facies.

\section{Conclusion.}

The functorial models of a landscape field proposed make it possible to differently consider the geographical envelope and regularities of its formation. Interrelations (morphisms) of different parts of geographical complex are the basic parameter of a landscape field. The existence of the unity between intercomponent, spatial and temporal functors of the geographical envelope, which form the entire Earth's landscape field, has been grounded. However, spatial and temporal changes are not parts of one category. Each temporal inetrval forms a category and different time periods

\footnotetext{
${ }^{\dagger}$ Distribution of system elements in decreasing order of frequency of their occurrence.
} 
form a functor. It means that temporal and spatial changes of geocomplexes occur within the integrated system but revealed at different levels.

The method of the Jacobian determinant makes it possible to prove the existence of intercomponent, diachronic and diamer categories and functors in nature and to restore the structure of a landscape field in the form of boundaries of functional-homogeneous domains. Congruent modelling provides a possibility of identifying the parameters of categories and functors and belonging of an object to a certain functor and of modelling the hierarchy of landscape complexes. All these factors allow the use of landscape mapping: the boundaries of landscape units are identified with the Jacobian determinant, parameters of congruent functions depend on their typological belonging.

The category-functorial approach permits reconsidering the problems of discreteness and continuity of the Earth's landscape envelope and determination of properties and parts of a landscape complex: a continuity of the field of characteristics of the landscape components transforms to a discreteness of the functions of their connection, when a type of connection changes on the boundary of a functionally homogenous unit. The parts of complexes are not equilibrium. They are changeable and variable, but the structural and functional similarity of parts of the unity and their connectedness are always retained. Notwithstanding a great variety of intra-system connections of geocomplexes, they are all functionally similar and produce a spatial organization of the planet in the form of a landscape field, which is a necessary and sufficient condition for sustainable existence of the Earth's geographical envelope. The fulfilment of this condition provides sustainable development in transformations of the territory, i.e. the creation of geotechnical complexes as an integral part of a landscape field.

How should the conservation of the natural balance be achieved during the creation of geotechnical complexes? Which regularities does the existence of disturbed transitional complexes conform to? The solution of these problems calls for further investigations.

\section{References}

[1] A.D. Armand. Self-organization and self-regulation of geographical systems. Nauka, Moskow, 1988.

[2] D.L. Armand Field theory and problems of identification of geosystems. Problems of Geography, 1975, 92-106.

[3] D.L. Armand Geographical environment and rational use of natural resources.Nauka,Moskow, 1983.

[4] I.C. Baianu Natural transformation models in molecular biology. Illinois, USA, 2004.

[5] A.M. Berlyant Maps of phenomenon relationships and their application in geographical studies.Bulletin of Moscow University, Series V, Geography, No. 1, 1972, 21-30.

[6] I. Bukur, A. Delyanu. Introduction to theory of categories and functors. Mir, Moskow, 1972. 
[7] A.K. Cherkashin. Mathematical analysis of dynamics of taiga facies.Structure and Dynamics of Geosystems. Nauka, Novosibirsk, 1979, 121-132.

[8] A.K. Cherkashin. Polysystem modelling. Nauka, Novosibirsk, 2005. 280 p.

[9] A.K. Cherkashin , E.A. Istomina Peculiarities of modelling of the Earth's landscape field. Landscape Analysis for Sustainable Development. Theory and Applications of Landscape Science in Russia. Eds. K.N. Dyakonov, N.S. Kasimov, A.V. Khoroshev, A.V. Kushlin. Alex Publisher, Moscow, 2007, 80-93.

[10] V.A. Chervyakov. Field conception in contemporary cartography. Novosibirsk, 1978.

[11] V.A. Chervyakov Peculiarities of using field maps in geography. Field maps of dynamics and interrelations of phenomena. Irkutsk, 1980, 7-15.

[12] L.E. Elsgolts. Introduction to theory of differential equations with a perturbation argument. Nauka, Moskow, 1964.

[13] Geoinformation modelling and mapping of landscape complexes of Pribaikalye. Abstract of Ph.D. thesis by E.A.Istomina. Izd-vo Institute of Geography SB RAS, Irkutsk, 2006.

[14] A.A. Grigoryev. Regularities of structure and development of geographical environment, Moskow, 1966.

[15] A.G. Isachenko.Landscape science yesterday and today. Izvestiya RGO, 2006, No 5, 1-20.

[16] A.G. Isachenko. Theory and methodology of geographical science. Academy, Moskow, 2004.

[17] E.A. Istomina, A.K. Cherkashin. Mathematical models of geographical complex and their application in analysis of space information Izvestiya RAN, Ser. Geogr, 2005, No. 2, - 103 113.

[18] S.V. Kalesnik. General geographical regularities of the Earth. Moskow, 1970.

[19] T.V. Keiko, A.K. Cherkashin. Theory of presentation of geoimages for geoindication. Geoinformation Science - 2000: Proceedings of International Scientific Conference, Izd-vo TSU, Tomsk, 2000, 26-29.

[20] A.V. Khoroshev, G.M. Aleshchenko. Characteristic space of intercomponent relationships in landscape. Bulletin of Moscow University, Series 5, Geography, 2008, No. 1.

[21] G.D. Kostinsky. Geographical matrix of space.Izvestiya RAN, Ser. Geogr., 1997, No. 5, - P. 16-34.

[22] A.A. Krauklis. Problems in experimental landscape studies. Nauka, Novosibirsk, 1979.

[23] Landscape-interpretation mapping. Nauka, Novosibirsk, 2005. 
[24] Landscapes of South-Eastern Siberia. Map scale 1:1 500 000. V.S Mikheev, V.A. Ryashin V.A. GUGK, Moskow, 1977.

[25] A.P. Levich. Theory of sets, language of theory of categories and their application in theoretical biology. Izd-vo Moscow Univ., Moskow, 1982.

[26] Levich A.P., Nosov V.N., Smirnov N.A. Theoretical-category model of structure of ecological community taking into account external factors // Man and Biosphere. M.: Izd-vo Moscow Univ., 1983, Issue 8. - P. 134-139.

[27] A.P. Levich,A.V. Solovyev. Category-functorial modelling of natural systems.Analysis of system on the XXI century threshold. Intellect, Moskow, 1997, 66-78. http://www.chronos.msu.ru/RREPORTS/levich_kategorno/levich_kategorno.htm

[28] Maps of density fields in geographical studies. Irkutsk, 1978.

[29] Maps of dynamics fields and relationships of phenomena. Irkutsk, 1980.

[30] Yu.P. Mikhailov. Density field maps in geographical resource management. Density field maps in geographical studies. Irkutsk, 1978, 16-32.

[31] E. Neef. Theoretical fundamentals of landscape science. Progress, Moskow, 1974.

[32] V.A. Nikolaev. Landscape space-time (methodological aspects).Bulletin of Moscow Univ, Ser. 5, Geogr., 1989, No. 2.

[33] B.Pareigis. Categories and functors. Acad. Press, N.Y., 1970.

[34] V.S. Preobrazhensky. Landscape studies. Nauka, Moskow, 1966.

[35] V.S. Preobrazhensky, T.D. Alexandrova, L.V. Maximova. Geography in the changing world. XX century. IG RAN, Moskow, 1997.

[36] Remote studies and mapping of structure and dynamics of geosystems. Izd-vo Institute of Geography SB RAS, Irkutsk, 2002.

[37] R. Rosen. Foundation of mathematical biology. Leipzig, 1972.

[38] Yu.A. Shreider, A.A. Sharov. Systems and models. Radio and Communication, Moskow, 1982.

[39] V.B. Sochava. Introduction to studies of geosystems. Nauka, Novosibirsk, 1978.

[40] A.M. Trofimov, E.I. Igonin. Conceptual patterns of modelling in geography. Development of basic ideas and techniques of mathematization and formalization in geography. Izd-vo "Matbugat yorty", Kazan, 2001. 
[41] A.M. Trofimov, A.G. Stepin, G.A. Stepin. Spatial autocorrelation as the method of quality assessment of territorial organization. Dep. In VINITI, No. 13.00.00, 638-V00.

[42] S.Yu. Zheltov, A.V. Sibiryakov. Method of subpixel correlation for extremely accurate identification of appropriate points of stereoimages.Recent State and Prospects of Development of Geodesy, Phototopography, Cartography and Geoinformation Systems. Geodesy and Cartography. Moscow, 1997, No. 2.

[43] A.V. Zhirmunsky, V.I. Kuzmin. Critical levels of development of natural systems. Nauka, Leningrad, 1990.

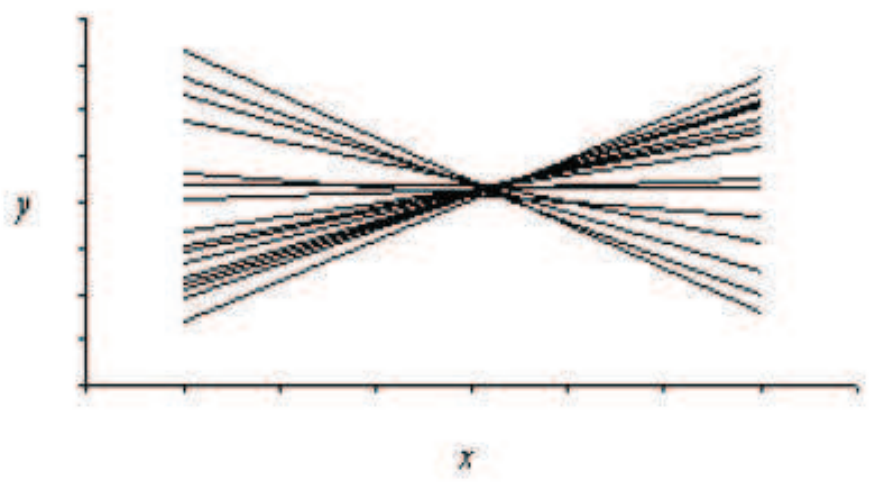

Figure 1: Linear congruence

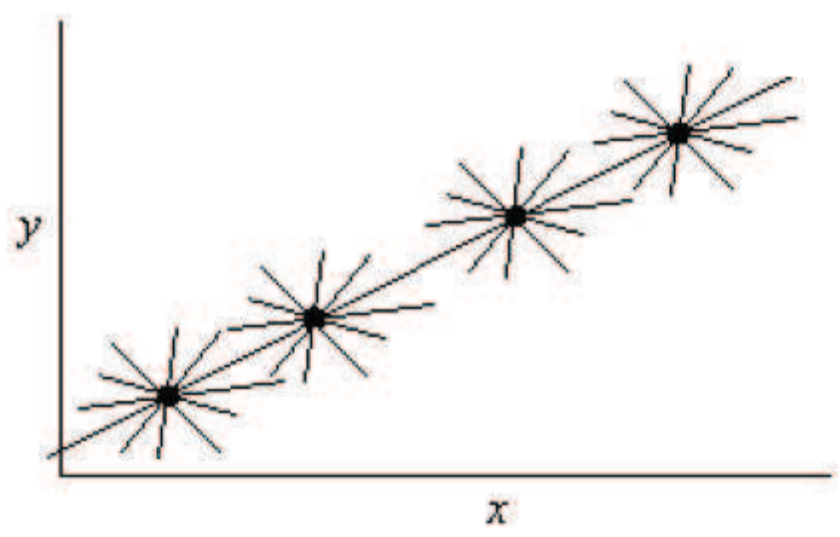

Figure 2: Linear complex 


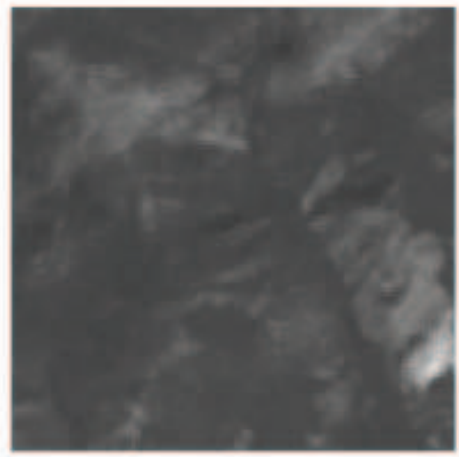

a

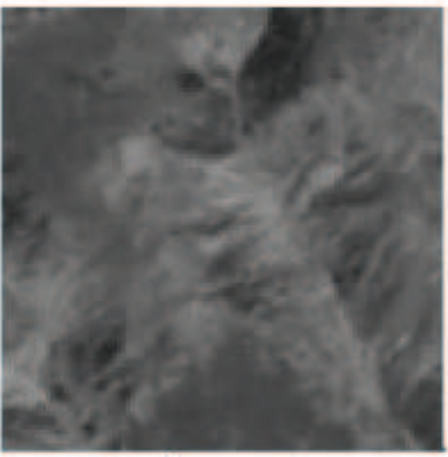

b

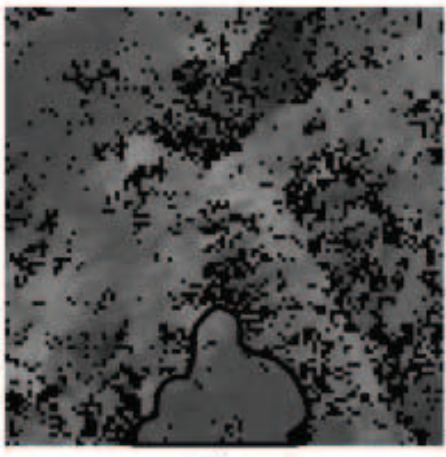

c

Figure 3: a, b - original images, green and reflected infrared channel, c - calculations by Jacobian overlapped on the original image $1 \mathrm{~b}$ (black contour - homogeneous unit) (explanatory notes to text)

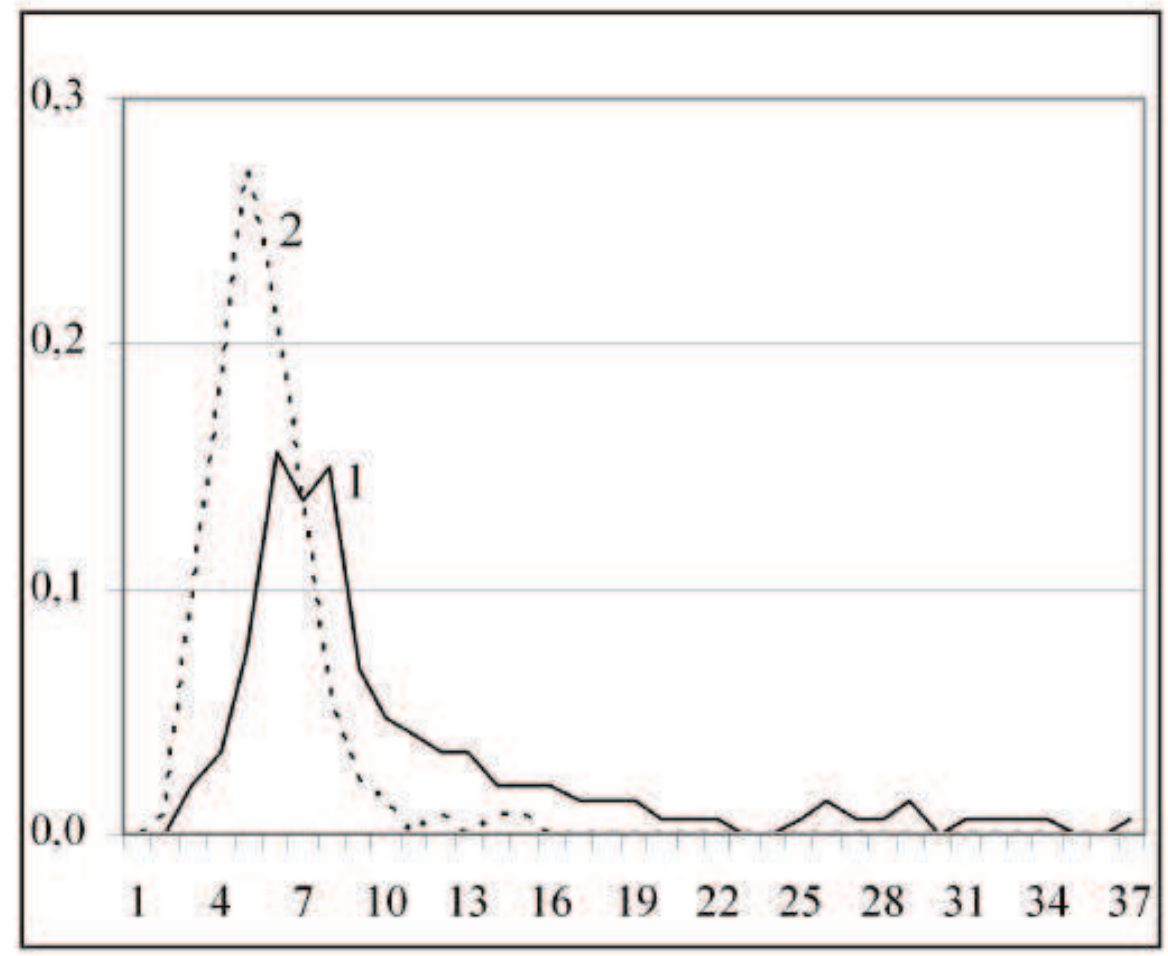

Figure 4: Frequency pixel distribution in the image of infrared (row 1) and red (row 2) channels inside homogenous unit 


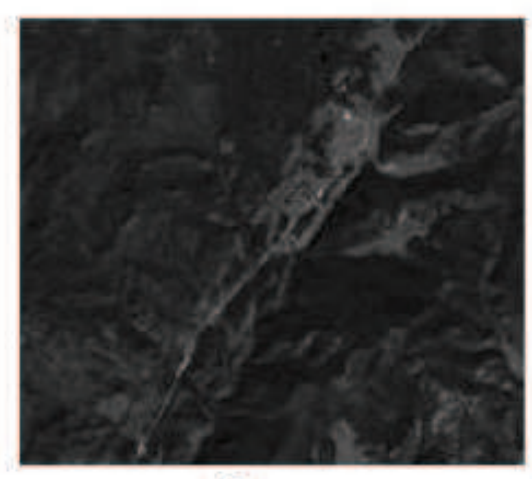

a

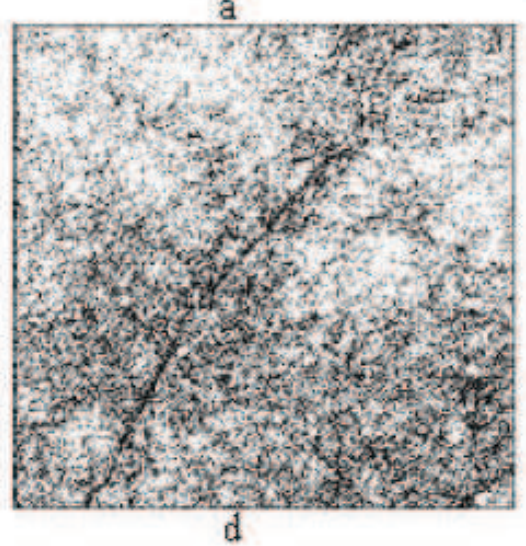

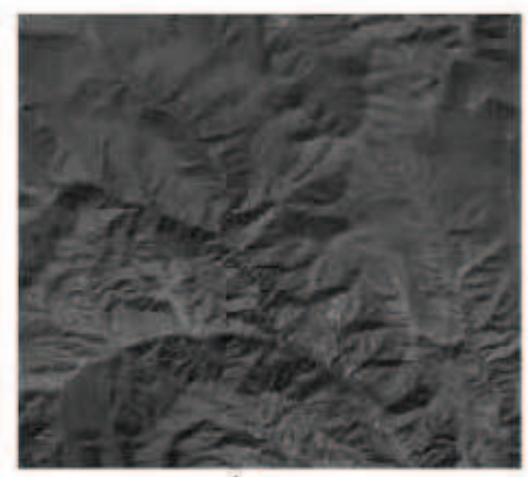

b

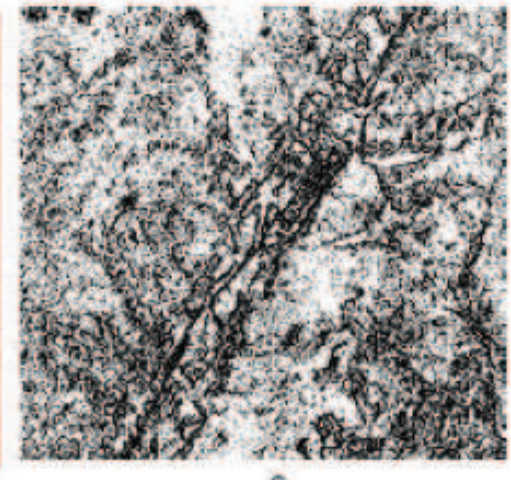

c

Figure 5: Boundary detection of homogenous areas in different images: a - Landsat image, red channel on the territory of the Primorsky Ridge; b - the same on the territory of the Kunlun Mountain system. Marked boundaries: $\mathrm{c}-$ combination of images taken at different time; $\mathrm{d}-$ combination of images a and $b$. 